\title{
Double Constrained NMF for Partial Multi-View Clustering
}

\author{
Bin Qian*, Xiaobo Shen*, Yanyang $\mathrm{Gu}^{\dagger}$, Zhenmin Tang* and Yuhua Ding* \\ * School of Computer Science and Engineering, Nanjing University of Science and Technology \\ Nanjing, 210094, China \\ †School of Information and Communication Technology, Griffith University \\ Nathan, QLD 4111, Australia
}

\begin{abstract}
Many problems in computer vision involve data sets with multiple views where observations are represented by multiple sources of features. To integrate information from multiple views in the unsupervised setting, multi-view clustering algorithms have been developed to cluster multiple views simultaneously. However, most of these algorithms only consider the situation that each example appears in all views, but can not deal with the case that the example may miss partial views data, which is often occurred in real applications, i.e., when a robot is investigating, the data may not be captured completely due to certain sensor fault. In this paper, we present a nonnegative matrix factorization method for partial multi-view clustering, which incorporates the cluster similarity and manifold preserving constraints into the unified framework. The basic principle of our proposed double constrained NMF (DCNMF), is pushing clustering solution of the same example in different views towards a common membership matrix, and meanwhile keeping the latent geometric structure of instances in the same view. Moreover, we develop the corresponding optimization scheme for our proposed method. Experiments on two-view datasets demonstrate the advantages of our proposed approach.
\end{abstract}

\section{INTRODUCTION}

In many real world application domains, multi-modal data, e.g., sound and images from the same video, text and pictures from the same webpage is prevalent in practice. How to deal with such multi-modal or multi-view data simultaneously is a fundamental and practical problem [1]. Although each individual view can be sufficient for characterizing the data object, the multiple views often contain complementary information to each other, which may help to alleviate the difficulty of a giving learning problem. Recently, multi-view clustering, which can exploiting multiple views to effectively learn from unlabeled data, has been widely concerned [2], [3].

A number of multi-view clustering methods have been proposed. Among them, there are two main clustering categories: spectral based or subspace based. Roughly speaking, spectral based methods are extensions of single-view clustering methods with extra similarity measures. MinDisSC [4] is one of the earliest approaches. This method creates a bipartite graph and adopts the 'minimizing-disagreement' criterion for spectral clustering. Co-training Spectral clustering (CtSC) [5] has a flavor of co-training, which searches for the clusterings that agree across all the views. In [6], a Co-regularized Spectral clustering ( $\mathrm{CrSC}$ ) method is proposed, which co-regularizes the clustering hypotheses that corresponding data points in each view should have same cluster membership and two coregularization schemes are exploited to accomplish this goal.
A typical subspace based method is Canonical Correlation Analysis (CCA) [7], which has been proposed as a mean for both dimension reduction [8] and multi-view subspace learning [9], [10]. Generally, CCA based multi-view clustering methods first project multi-view data into a common lowdimensional subspace and then apply any clustering algorithm such as K-means to learn the partition. Feature learning based approaches have also been reported [11], [12]. They combine feature selection strategy with extra structure constraints, such as group sparse constraint, to extract the most effective and irredundant features across the views.

In recent years, nonnegative matrix factorization (NMF) [13] has become a popular technique for data clustering, and it is reported to achieve competitive performance compared with most of the state-of-the-art unsupervised algorithms. NMF has received much attention because of its straightforward interpretability for applications, i.e., we can explain each observation as an additive linear combinations of nonnegative basis vectors, which accords with the cognitive process of human brain from the psychological and physiological studies [14]. Recently, NMF has been proposed as a subspace based multiview clustering method and achieve promosing results. In [15], a collective NMF (ColNMF) method is proposed for relational learning, which treats the multi-view clustering as a latent space searching problem, and decomposes each view data into two matrices, i.e., projection matrix and shared coefficient matrix, simultaneously. Liu et al. [16] propose multi-view clustering via joint nonnegative matrix factorization (MVNMF), which tries to search for a factorization that gives compatible clustering solutions across multiple views. However, in real tasks, it is often the case that each view suffers from some missing information, which results in many partial examples. The above multi-view clustering methods can not be naturally applied to the partial examples scene, and thus can not get a satisfactory clustering performance if discarding the partial examples directly. In order to solve this issue, a partial multiview clustering (PVC) method based on NMF is proposed [17]. PVC explicitly utilized the paired examples (example with full view data) as a bridge to combine partial examples (example with partial view data) information together and explore a latent subspace for clustering. However, PVC method neglects the relationship of instances in the same view and the shared latent subspace assumption is too strong, which may lead to poor performance when the attributes of these views are relatively distinct from each other.

Inspired with manifold [18] and shared subspace assump- 
tions, in this paper, we propose a double constrained NMF (DCNMF) method for partial multi-view clustering. In DCNMF, two constrains (manifold preserving and cluster similarity) are incorporated into the factorization process of NMF. The basic principle of our proposed approach is pushing clustering solution of the same example in different views towards a common membership matrix, and meanwhile keeping the latent geometric structure of instances in the same view. Thus the relationship between instances and examples are both considered in DCNMF, which substantially enhance the clustering performance. Moreover, a fast optimization scheme is developed for our proposed method. Experiments on synthetic and real world datasets validate the advantages of our DCNMF approach.

In the following we start with a brief review of some related work. Then, we propose our DCNMF approach and report the experimental results. Finally, we conclude the paper.

\section{RELATED WORK}

\section{A. $N M F$}

Given a non-negative matrix $X=\left[x_{1}, \cdots, x_{n}\right] \in R^{m \times n}$, where each vector $x_{i}$ represents a sample. NMF aims to seek two low-rank nonnegative matrices $U \in R^{m \times r}$ and $V \in R^{r \times n}$, where $r \ll \min (m, n)$. Such that the product of $U$ and $V$ can well approximate the original data matrix $X$. Thus, the objective function of NMF can be expressed as follow:

$$
\min _{U, V} F(U, V)=\frac{1}{2}\|X-U V\|_{F}^{2}, \quad \text { s.t. } U \geq 0, V \geq 0,
$$

where $\|\cdot\|_{F}$ denotes the matrix Frobenius norm. The most popular optimization method of NMF is the multiplicative updating algorithm proposed by Lee and Seung [13], which consists of two steps:

$$
U \leftarrow U . * \frac{X V^{T}}{U V V^{T}}, \quad V \leftarrow V . * \frac{U^{T} X}{U^{T} U V},
$$

where $(.)^{T}$ is the transpose of a matrix, .* denotes elementwise multiplication. It is proved that after a few iterations the objective function in equation (1) can converge to a local optimum [13]. In [19], a close relationship between Kmeans and NMF is presented. Suppose we do a K-means clustering on nonnegative data $X$ and obtain cluster centroids $U=\left(u_{1}, \cdots, u_{r}\right)$. Let $V$ denotes the cluster indicators, i.e., $v_{r i}=1$, if $x_{i}$ belongs to cluster $c_{r}, v_{r i}=0$, otherwise. We can write the K-means clustering objective function as

$$
F_{K}(U, V)=\sum_{i=1}^{n} \sum_{k=1}^{r} v_{k i}\left\|x_{i}-u_{k}\right\|_{F}^{2}=\|X-U V\|_{F}^{2} .
$$

We see that the K-means clustering objective can be alternatively viewed as an objective function of NMF if we relax the constraint by allowing $v_{k i}$ to range over values in $(0,+\infty)$.

\section{B. ColNMF for Multi-view Clustering}

For multi-view clustering task, a simple NMF based method is first presented in [15], which is called collective matrix factorization (ColNMF). Given a data set $X=\left\{\left(x_{i}^{(1)}, x_{i}^{(2)}, \cdots, x_{i}^{(q)}, y_{i}\right), i=1, \cdots, n\right\}$, where $x_{i}=$ $\left(x_{i}^{(1)}, x_{i}^{(2)}, \cdots, x_{i}^{(q)}\right)$ is the $i^{t h}$ example, $x_{i}^{(j)}$ is the instance

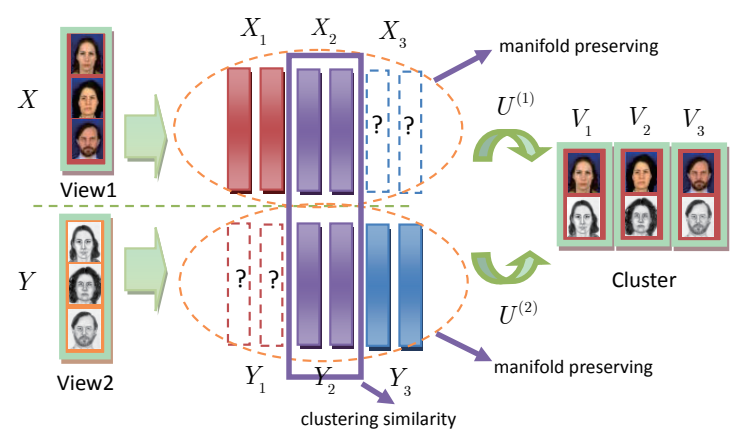

Fig. 1. Double constrained NMF model for partial multi-view clustering.

of the $i^{t h}$ example in the $j^{t h}$ view, $q$ is the number of views, and $y_{i}$ is its cluster label. ColNMF aims at clustering $x_{i}$ into it's corresponding cluster label $y_{i}$ through each single view's factorization with the shared coefficient matrix. The objective can be formulated as

$$
\begin{array}{r}
\min F\left(U^{(j)}, V\right)=\frac{1}{2} \sum_{j=1}^{q}\left\|X^{(j)}-U^{(j)} V\right\|_{F}^{2}, \\
\text { s.t. } U^{(j)} \geq 0, V \geq 0,
\end{array}
$$

where $X^{(j)}$ represents all the instances of the $j^{\text {th }}$ view. $U^{(j)}$ represents the projection matrix for the $j^{t h}$ view, and $V$ is the shared coefficient matrix. The above equation can be solved by iterative AO optimization: At each iteration, fixing $U^{(j)}, V$ is optimized; Then fixing $V, U^{(j)}$ is optimized independently.

\section{APPROACH}

In this section, we first describe the motivation of our approach and then give the formulation of double constrained NMF model for partial multi-view clustering. Finally the corresponding optimization algorithm is presented in detail.

\section{A. Motivation}

For the convenience of discussion, in this paper, assume that we are handling two-view data. The multi-view example set is given as

$$
\left[\begin{array}{l}
X \\
Y
\end{array}\right]=\left[\begin{array}{ccc}
X_{1} & X_{2} & X_{3} \\
Y_{1} & Y_{2} & Y_{3}
\end{array}\right]
$$

where $X=\left[X_{1}, X_{2}, X_{3}\right]$ and $Y=\left[Y_{1}, Y_{2}, Y_{3}\right]$ denote the instances of the first view and second view respectively. $X_{1} \in R^{d_{1} \times n_{1}}, X_{2} \in R^{d_{1} \times n_{2}}, X_{3} \in R^{d_{1} \times n_{3}}, Y_{1} \in R^{d_{2} \times n_{1}}$, $Y_{2} \in R^{d_{2} \times n_{2}}, Y_{3} \in R^{d_{2} \times n_{3}}$. In order to simulate the partial multi-view scene, we assume that the data of $Y 1$ and $X 3$ are missing. Thus $X_{1}$ and $Y_{3}$ are partial examples and $\left[X_{2} ; Y_{2}\right]$ are paired examples. The goal of our partial multi-view clustering method, same as ColNMF multi-view clustering, is to find their corresponding coefficient matrix $V_{1}, V_{2}$ and $V_{3}$, then cluster the examples into their corresponding clusters. As shown in Fig.1. The symbol '?' means the missing view data of partial examples. In order to explicitly explore the relationship of instances in the same view, a manifold assumption need to be considered in the multi-view clustering (red dotted ellipse in Fig.1): if two instances are close with each other in the high-dimension space, then there is a big chance that the two 
instances are still close after projection to the low-dimension space. Thus if we incorporate the manifold constraint into NMF model, we can obtain a more accurate projection matrix which can keep the intrinsic geometric structure of instances. On the other hand, although we lose the view data of some examples, we still have partial paired examples with full view data. Thus the shared subspace assumption for multi-view clustering presented in [9], [10], [15], [17] can still be hold for these paired examples (purple line box in Fig.1). However, the shared subspace constraint is too strong, which is not applicable in general cases. From Section 2, we known that the coefficient matrix after matrix factorization can reflect the cluster relationship of all examples, thus in this paper, we relax the hard shared subspace constraint and instead pushing the coefficient matrix of each view towards a common consensus, which has a clear physical meaning: the instances corresponding to the same example in different views should have a similar clustering solution under the NMF framework. Thus in our approach, two constraints reflecting different perspectives of information among the multi-view data, are embedded into NMF model, which will help to improve the cluster performance.

\section{B. Formulation}

A nearest neighbor graph can be constructed with the instances of each view. Denote the weight matrix of the graph as $E$. If $x_{i}$ is one of the k-nearest neighbors of $x_{j}$, then the weight is assigned as:

$$
E_{i j}= \begin{cases}1 & , \text { if } x_{i} \in \mathrm{N}_{p}\left(x_{j}\right) \text { or } x_{j} \in \mathrm{N}_{p}\left(x_{i}\right) \\ 0 & , \text { otherwise }\end{cases}
$$

It is apparent that when $x_{i}$ and $x_{j}$ are close, $E_{i j}$ is relatively big. According to the above analysis, once $x_{i}$ and $x_{j}$ are close, their new representations $v_{i}$ and $v_{j}$ in the new space should be close too. To achieve so, consider minimizing the following function:

$$
\begin{aligned}
\frac{1}{2} \sum_{i, j=1}^{n}\left\|v_{i}-v_{j}\right\|_{F}^{2} E_{i j} & =\sum_{i=1}^{n} v_{i}^{T} v_{i} D_{i i}-\sum_{i, j=1}^{n} v_{i}^{T} v_{j} E_{i j} \\
& =\operatorname{Tr}\left(V D V^{T}\right)-\operatorname{Tr}\left(V E V^{T}\right) \\
& =\operatorname{Tr}\left(V L V^{T}\right),
\end{aligned}
$$

where $\operatorname{Tr}($.$) represents the trace of a matrix, D$ is a diagonal matrix in which $D_{i i}=\sum_{j} E_{i j}$, and $L=D-E$. It is obvious that $L$ is symmetric. Take the first view as an example, incorporating this manifold preserving constraint term into the NMF leads to the cost function as follows:

$$
\begin{aligned}
\min F_{1}= & \left\|\left[X_{1}, X_{2}\right]-U^{(1)}\left[V_{1}, V_{2}\right]\right\|_{F}^{2} \\
& +\alpha\left[V_{1}, V_{2}\right] L^{(1)}\left[V_{1}, V_{2}\right]^{T} \\
& \text { s.t. } V_{1} \geq 0, V_{2} \geq 0,
\end{aligned}
$$

where $L^{(1)} \in R^{\left(n_{1}+n_{2}\right) \times\left(n_{1}+n_{2}\right)}$ denotes the Laplacian matrix for instances of the first view, which describes the manifold structure of both paired examples $X_{2}$ and partial examples $X_{1}$.

In order to explore the clustering similarity of paired examples, we first define a consensus matrix $V^{*}$, then the loss function to measure the disagreement between coefficient matrix $V$ and the consensus matrix $V^{*}$ can be given as

$$
\text { Dis }=\left\|V-V^{*}\right\|_{F}^{2} \text {. }
$$

In NMF model, the coefficient matrix $V$ reflects the cluster relationship, thus Eq. (9) is meaningful for clustering similarity measuring.

Combining Eq. (9) with the NMF model, we can derive the final objective of our proposed DCNMF:

$$
\begin{aligned}
\min F= & \sum_{i=1}^{2}\left\|\tilde{X}^{(i)}-U^{(i)} \tilde{V}^{(i)}\right\|_{F}^{2}+\alpha \operatorname{Tr}\left(\tilde{V}^{(i)} L^{(i)}\left(\tilde{V}^{(i)}\right)^{T}\right) \\
& +\beta\left\|V_{2}^{(i)}-V^{*}\right\|_{F}^{2} \\
& \text { s.t. } V^{*} \geq 0, \tilde{V}^{(1)}=\left[V_{1}, V_{2}^{(1)}\right] \geq 0 \\
& \tilde{V}^{(2)}=\left[V_{3}, V_{2}^{(2)}\right] \geq 0, U^{(i)} \geq 0
\end{aligned}
$$

where $\tilde{X}^{(1)}=\left[X_{1}, X_{2}\right], \tilde{X}^{(2)}=\left[Y_{3}, Y_{2}\right]$. The parameters $\alpha$ and $\beta$ control the weights of manifold preserving and clustering similarity constraints, respectively. Note that the objective in Eq. (10) is essentially different with the simple concatenation of each view's factorization due to the consensus matrix $V^{*}$.

\section{Optimization}

To address the optimization in Eq. (10), which is convex in latent representations $V_{1}, V_{2}^{(1)}, V_{2}^{(2)}$, and $V_{3}$ given the basis matrix $U^{(i)}$ and vice versa, but not jointly convex in both, we propose a fast iterative update procedure based on multiplicative updating. Firstly, the basis matrixs and coefficient matrixs are initialized by the initialization step and then the following three steps are repeated until convergence:1) minimizing $F$ over $V_{1}, V_{2}^{(1)}, V_{2}^{(2)}$, and $V_{3}$ with fixed $U^{(i)}$ and $V^{*} ; 2$ )minimizing $F$ over $U^{(i)}$ with fixed $V_{1}, V_{2}^{(1)}, V_{2}^{(2)}$, and $V_{3}$; 3)minimizing $F$ over $V^{*}$ with fixed $V_{2}^{(1)}$ and $V_{2}^{(2)}$.

1) Initialization: In order to speed up the convergence, in this paper, we learn the initial value of each variable matrix rather than random allocation. The optimization equation is shown as below

$$
\begin{aligned}
\min F_{i}= & \left\|\tilde{X}^{(i)}-U^{(i)} \tilde{V}^{(i)}\right\|_{F}^{2}+\alpha \operatorname{Tr}\left(\tilde{V}^{(i)} L^{(i)}\left(\tilde{V}^{(i)}\right)^{T}\right) \\
& \text { s.t. } \forall i, \tilde{V}^{(i)} \geq 0, U^{(i)} \geq 0 .
\end{aligned}
$$

Note that Eq. (11) is actually equal with the objective of GNMF [18]. Thus the corresponding update rules of $U^{(i)}, \tilde{V}^{(i)}$ can be directly given as below:

$$
\begin{gathered}
U^{(i)} \leftarrow U^{(i)} \cdot * \frac{\tilde{X}^{(i)}\left(\tilde{V}^{(i)}\right)^{T}}{U^{(i)} \tilde{V}^{(i)}\left(\tilde{V}^{(i)}\right)^{T}} \\
\tilde{V}^{(i)} \leftarrow \tilde{V}^{(i)} \cdot * \frac{\left(U^{(i)}\right)^{T} \tilde{X}^{(i)}+\alpha \tilde{V}^{(i)} E^{(i)}}{\left(U^{(i)}\right)^{T} U^{(i)} \tilde{V}^{(i)}+\alpha \tilde{V}^{(i)} D^{(i)}},
\end{gathered}
$$

After convergence, we normalize $U^{(i)}, \tilde{V}^{(i)}$ according to the next equation [18]:

$$
v_{r j} \leftarrow \frac{v_{r j}}{\sqrt{\sum_{j} v_{r j}^{2}}}, \quad u_{i r} \leftarrow u_{i r} \sqrt{\sum_{j} v_{r j}^{2}} .
$$

Then the initial value of $V^{*}$ can be obtained by

$$
V^{*}=\frac{V_{2}^{(1)}+V_{2}^{(2)}}{2} \text {. }
$$


2) Minimizing $F$ over $V_{2}^{(1)}, V_{2}^{(2)}$, with fixed $V_{1}, V_{3}, V^{*}$, $U^{(1)}, U^{(2)}$ : We only care about terms that are relevant to $V_{2}^{(1)}$, Eq. (10) reduces to minimize:

$$
\begin{aligned}
\min _{V_{2}^{(1)}} & \left\|X_{2}-U^{(1)} V_{2}^{(1)}\right\|_{F}^{2}+\alpha \operatorname{Tr}\left[V_{2}^{(1)} L_{d}^{(1)}\left(V_{2}^{(1)}\right)^{T}\right. \\
& \left.+V_{2}^{(1)} L_{c}^{(1)}\left(V_{1}\right)^{T}+V_{1} L_{b}^{(1)}\left(V_{2}^{(1)}\right)^{T}\right]+\beta\left\|V_{2}^{(1)}-V^{*}\right\|_{F}^{2}, \\
& \text { s.t. } V_{2}^{(1)} \geq 0,
\end{aligned}
$$

where $L_{a}^{(1)}, L_{b}^{(1)}, L_{c}^{(1)}, L_{d}^{(1)}$ represents the first $n_{1}$ rows and first $n_{1}$ columns, the first $n_{1}$ rows and last $n_{2}$ columns, the last $n_{2}$ rows and first $n_{1}$ columns, the last $n_{2}$ rows and last $n_{2}$ columns of $L_{1}$, respectively. By taking the first order derivative of Lagrange $F$ over $V_{2}^{(1)}$, we can get the following update rule for $V_{2}^{(1)}$ :

$$
\begin{aligned}
& V_{2}^{(1)} \leftarrow V_{2}^{(1)} \cdot * \\
& \frac{\left(U^{(1)}\right)^{T} X_{2}+\alpha\left(V_{2}^{(1)} E_{d}^{(1)}+V_{1} E_{b}^{(1)}\right)+\beta V^{*}}{\left(U^{(1)}\right)^{T} U^{(1)} V_{2}^{(1)}+\alpha\left(V_{2}^{(1)} D_{d}^{(1)}+V_{1} D_{b}^{(1)}\right)+\beta V_{2}^{(1)}} .
\end{aligned}
$$

The update rule of $V_{2}^{(2)}$ can be derived similarly with $V_{2}^{(1)}$.

3) Minimizing $F$ over $V_{1}, V_{3}$, with fixed $V_{2}^{(1)}, V_{2}^{(2)}, U^{(1)}$, $U^{(2)}$ : We only care about terms that are relevant to $V_{1}$, Eq. (10) reduces to minimize:

$$
\begin{aligned}
\min _{V_{1}} \| & X_{1}-U^{(1)} V_{1} \|_{F}^{2}+\alpha \operatorname{Tr}\left[V_{1} L_{a}^{(1)} V_{1}^{T}+V_{2}^{(1)} L_{c}^{(1)} V_{1}^{T}\right. \\
& \left.+V_{1} L_{b}^{(1)}\left(V_{2}^{(1)}\right)^{T}\right] \text {, s.t. } V_{1} \geq 0 .
\end{aligned}
$$

Thus we can derive the update rule of $V_{1}$ as below:

$$
V_{1} \leftarrow V_{1} \cdot * \frac{\left(U^{(1)}\right)^{T} X_{1}+\alpha\left(V_{1} E_{a}^{(1)}+V_{2}^{(1)} E_{c}^{(1)}\right)}{\left(U^{(1)}\right)^{T} U^{(1)} V_{1}+\alpha\left(V_{1} D_{a}^{(1)}+V_{2}^{(1)} D_{c}^{(1)}\right)} .
$$

The update rule of $V_{3}$ can be derived similarly with $V_{1}$.

4) Minimizing $F$ over $U^{(1)}, U^{(2)}$, with fixed $V_{2}^{(1)}, V_{2}^{(2)}$, $V_{1}, V_{3}$ : We only care about terms that are relevant to $U^{(1)}$, Eq. (10) reduces to minimize:

$$
\min F_{1}=\left\|\tilde{X}^{(1)}-U^{(1)} \tilde{V}^{(1)}\right\|_{F}^{2} .
$$

Comparing with Eq. (11), we can see that the update rules of $U^{(1)}$ and $U^{(2)}$ are exactly same with Eq. (12).

5) Minimizing $F$ over $V^{*}$, with fixed $V_{2}^{(1)}, V_{2}^{(2)}$ : Take the derivative of the objective function $F$ in Eq. (10) over $V^{*}$, we can get the same update rule with Eq. (15) for $V^{*}$.

The complete procedure is summarized in Algorithm 1. Note that once we obtain the latent matrix $V^{*}, V_{1}, V_{3}$, then the cluster label can be computed by K-means.

\section{EXPERIMENTS}

In this section, we conduct extensive experiments to evaluate the proposed DCNMF algorithm on synthetic data and real world data. To evaluate the effectiveness and efficiency, we present quantitative evaluations of the proposed DCNMF and compare it with some related approaches: CCA [7], MinDisSC [4], CrSC [6], ColNMF [15] and PVC [17]. Additionally, NMF with the data of each single view (SingleView1,

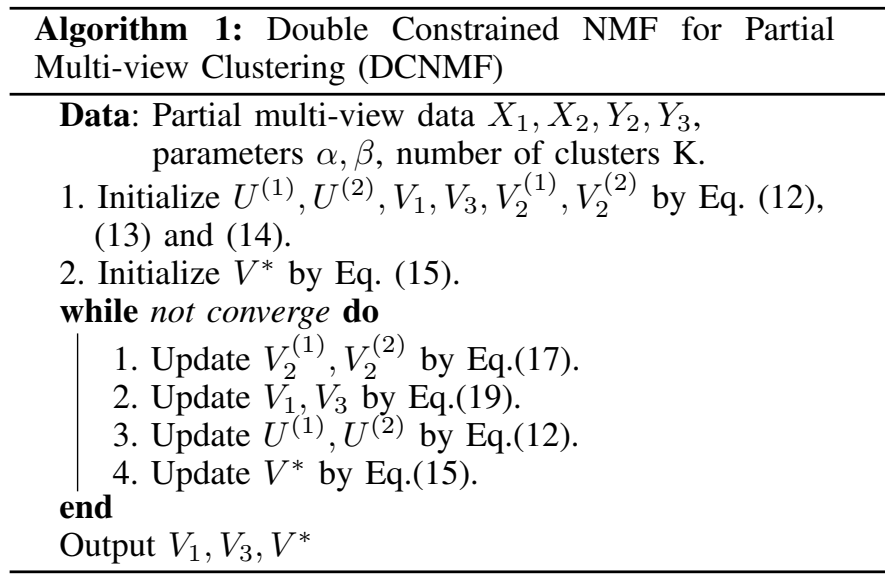

SingleView2) is adopted as two baselines in comparison. We utilize K-means to cluster the data points for subspace based methods and set the number of clusters to be the true number of classes. The clustering performance is evaluated by clustering Accuracy (AC) and Normalized Mutual Information (NMI) measures, which are the standard measures widely used for clustering [18]. To simulate the partial view setting, we randomly select a fraction of examples to be partial examples and denote $\mathrm{Per}$ as the ratio of partial examples. For each dataset, the $P e r$ is set by $\{0,0.1, \cdots, 0.7\}$. Such process is repeated 30 times and the average results are recorded. Note that except PVC and our proposed DCNMF, all the compared methods can not handle partial examples. For a fair comparison, a matrix completion method [21] is firstly adopted to fill in the missing information for these methods.

\section{A. Datasets}

One synthetic and three real world datasets are used in the experiment. Among the three real world datasets, the first two are text data [22], and the last one is handwritten digit data [23]. The important statistics of them are summarized in Table. I.

TABLE I. STATISTICS OF THE FOUR DATASETS

\begin{tabular}{ccccc}
\hline \hline Data Set & Size & Cluster & View1 & View2 \\
\hline Synthetic & 1200 & 3 & 3 & 3 \\
Texas & 187 & 5 & 187 & 1703 \\
Washington & 230 & 5 & 230 & 1703 \\
Digit & 2000 & 10 & 76 & 240 \\
\hline \hline
\end{tabular}

1) Synthetic dataset: Our synthetic experiment is designed on a toy two-view data: 400 points are generated for each cluster of each view by Gaussian mixture model. In either view, three of the randomly picked clusters centers are highly overlapped and therefore difficult to distinguish. As shown in Fig. 2(a) (b).

2) Texas dataset: The Texas dataset contains 187 documents over the 5 labels (student,project,course,staff,faculty). It is made of 2 views (content,cites) on the same documents. The documents are described by 1703 words in the content view, and by the 187 links between them in the cites view. 


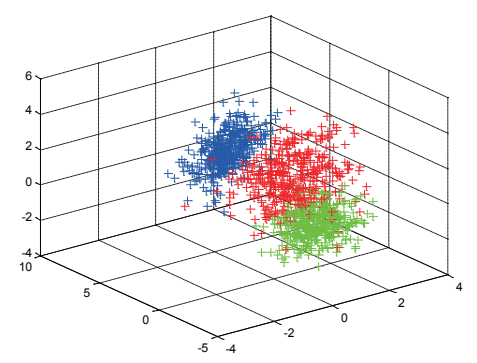

(a)

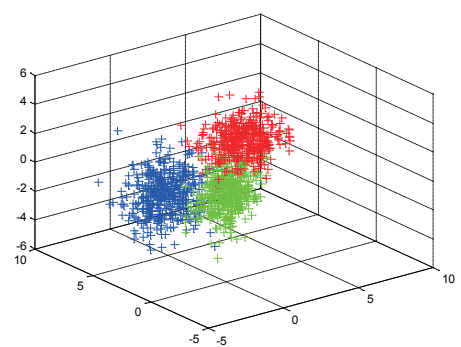

(b)

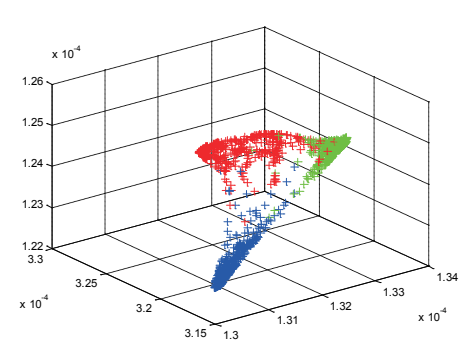

(c)

Fig. 2. Synthetic data example. (a) Original data of View1; (b) Original data of View2; (c) The latent representation data obtained by our proposed DCNMF

3) Washington dataset: The Washington dataset contains 230 webpages from Washington University. The webpages are distributed over five classes and it is made of 2 views (content,cites) on the same documents. The documents are described by 1703 words in the content view, and by the 230 links between them in the cites view.

4) Digit dataset: This dataset consists of features of handwritten numerals $\left({ }^{\prime} 0^{\prime}, \ldots,{ }^{\prime} 9^{\prime}\right)$ extracted from a collection of Dutch utility maps. 200 patterns per class (for a total of 2000 patterns) have been digitized in binary images, with view 1 being the 76 Fourier coefficients and view 2 being the 240 pixel averages in $2 \times 3$ windows.

\section{B. Results}

In synthetic experiment, Fig. 2(c) shows the corresponding latent representation matrix obtained by our proposed DCNMF when $P e r=0$. We can obviously observe that the distribution of each cluster is more compact and the distance between each cluster centroid is larger in Fig. 2(c). DCNMF considers to preserve the local affinity structure of each view and keep the cluster similarity of each example simultaneously. Thus, DCNMF can make the points of each cluster compact and explicitly integrate the information of the multi-view data through the paired examples.

Fig. 3 shows the clustering performance of different algorithms on all the four datasets when Per varying from 0 to $70 \%$ with an interval of $10 \%$. From Fig. 3 we can see that, for all four data sets, comparing the two NMF clustering results on each single view (SingleView1 and SingleView2), DCNMF always performs the best, which validates that our proposed method can explicitly integrate the information among two views and help to promote the final cluster performance.

When the partial example ratio Per is 0, the data set actually comes to the traditional multi-view data that each example appears in all views. In such case, DCNMF is also able to perform better than most baselines except for MinDisSC and $\mathrm{CrSC}$ in the synthetic experiment. One of the possible reasons is that the two views are generated independently with Gaussian mixture model, which is pretty suitable for covariance calculation based MinDisSC and CrSC. On the other hand, although MinDisSC and CrSC can get higher AC and NMI in Fig. 3(a), the performances of these two method drop sharply when increase Per, which illustrates that these two methods can not explicitly deal with the case of partial examples.
In real world datasets, the partial based multi-view cluster methods, PVC and our proposed DCNMF, perform much better than other NMF based methods. Specifically, PVC and DCNMF split the data into partial examples and paired examples, and then deal with them separately. Such a process has two advantages: (1) when aiming to find the latent structure of multi-view data, partial examples will not pollute the paired examples. If we deal with the case just as complete multiview data, like ColNMF, the partial examples will influence the final cluster result due to its incomplete information. (2) the partial examples can devote its own effective information to the process of multi-view cluster. However, in PVC, the information between paired examples and partial examples is totally neglected, which may limit the performance. Our proposed DCNMF incorporates the manifold structure into NMF which explores the latent information among all the instances of each view. Thus from the perspective of information utilization, our method is better than PVC, which accords with the experimental results.

\section{Parameters analysis}

In this subsection, we show how the regularization parameters $\alpha$ and $\beta$ can influence the performance of clustering. For our proposed DCNMF, we need to specify the trade-off parameters $\alpha, \beta$ and the number of nearest neighbor $k$, respectively. Here, we empirically set $k$ as 5 in all databases. Thus, we only explore the influences of $\alpha, \beta$ on the clustering performance. Specifically, considering the convenience of comparison, we set the partial example ratio Per as 0.3. We search the optimal parameters from a wide range of $\left[10^{-3}, 10^{2}\right]$. Fig. 4 shows the AC performances of DCNMF for varying the parameters $\alpha$ and $\beta$. As we can see, the performances of DCNMF are relatively stable and get the highest $\mathrm{AC}$ when the parameter $\alpha$ varies from 0.1 to 1 and $\beta$ varies from 1 to 10 on all datasets.

\section{CONCLUSiON}

In this paper, we introduce a novel algorithm for partial multi-view clustering based on nonnegative matrix factorization. In order to efficiently learn the underlying manifold and clustering structure embedded in multiple views, we require the close instances in the same view still keep close after factorization and meanwhile coefficient matrices learnt from paired examples are regularized towards a common cluster structure. To achieve this, we develop a matrix factorization algorithm to incorporate not only individual manifold constraint for each view but also cluster inconsistency constraint between 


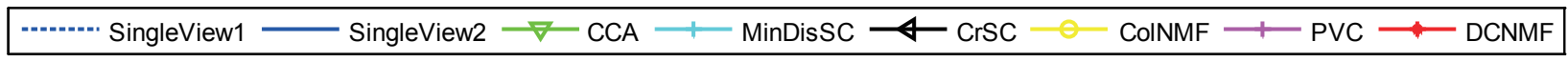
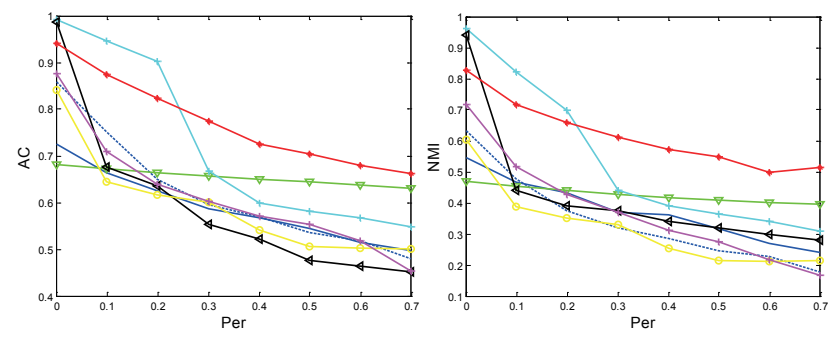

(a)
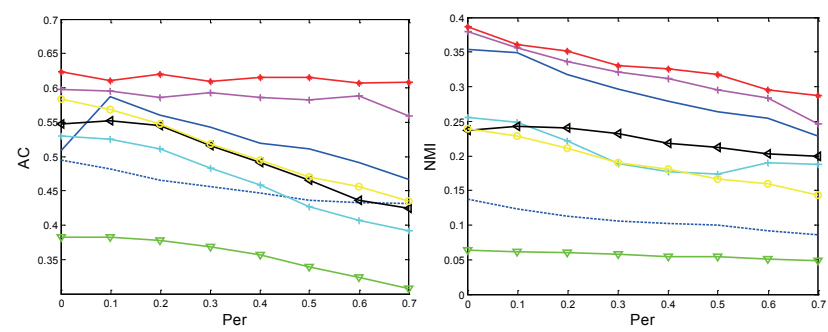

(c)
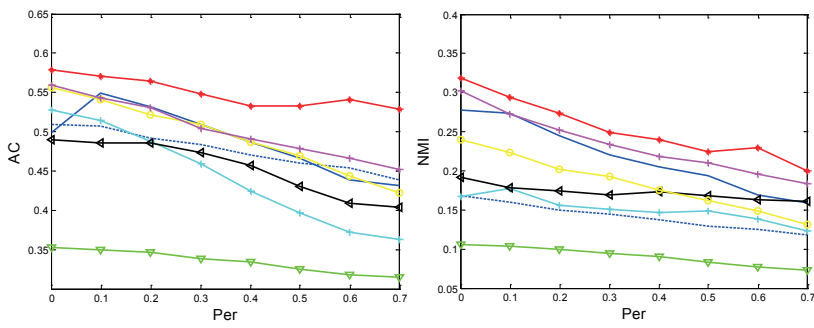

(b)
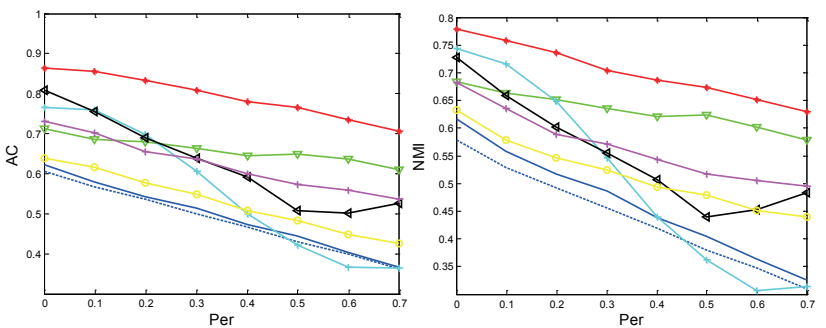

(d)

Fig. 3. The AC and NMI results for the four data sets. (a) Synthetic dataset; (b) Texas dataset; (c) Washington dataset; (d) Digit dataset

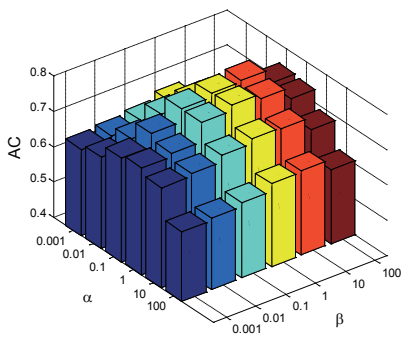

(a)

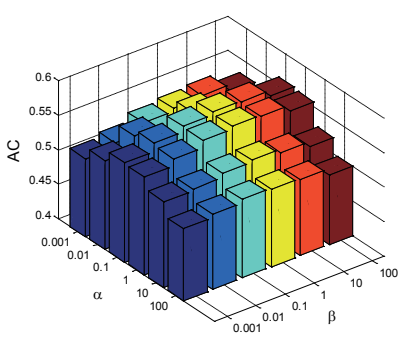

(b)

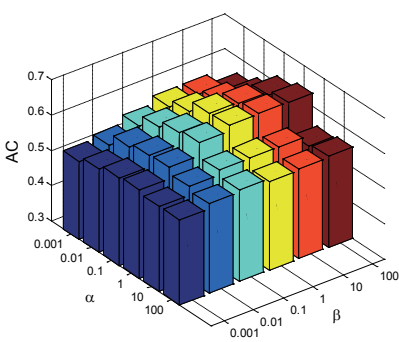

(c)

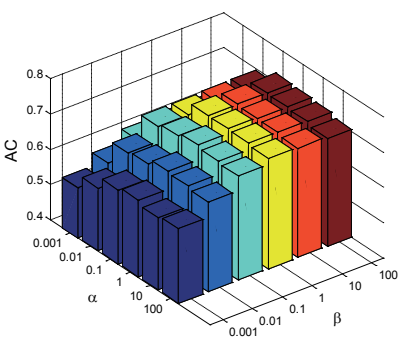

(d)

Fig. 4. The AC performances with the varied parameters $\alpha$ and $\beta$. (a) Synthetic dataset; (b) Texas dataset; (c) Washington dataset; (d) Digit dataset

each views coefficient matrix and the consensus. Moreover, we design an effective iterative procedure for the optimization problem. Experiments on both synthetic and three real world datasets demonstrate that the proposed method outperforms other state-of-the-art methods.

\section{ACKNOWLEDGMENT}

This research is supported by the grants of Natural Science Foundation of Jiangsu Province of China (Grant No. BK20140794) and Fundamental Research Funds for the Central Universities (Grant No.30916011326).

\section{REFERENCES}

[1] A. Torralba, K. P. Murphy, and W. T. Freeman. Sharing visual features for multiclass and multiview object detection. IEEE Transactions on Pattern Analysis and Machine Intelligence, 29(5):854-869, 2007.

[2] S. Bickel, and T. Scheffer. Multi-View Clustering. IEEE International Conference on Data Mining, 4:19-26, 2004.

[3] S. Sun. A survey of multi-view machine learning. Neural Computing and Applications, 23(7-8):2031-2038, 2013.

[4] V. R. de Sa. Spectral clustering with two views. ICML Workshop on Learning with Multiple Views, 20-27, 2005.
[5] A. Kumar, and H. Daum. A co-training approach for multi-view spectral clustering. Proceedings of the 28th International Conference on Machine Learning, 393-400, 2011.

[6] A. Kumar, p. Rai, and H. Daum. Co-regularized multi-view spectral clustering. Advances in neural information processing systems, 14131421, 2011.

[7] L. Sun, S. Ji, and J. Ye. Canonical correlation analysis for multilabel classification: A least-squares formulation, extensions, and analysis. IEEE Transactions on Pattern Analysis and Machine Intelligence, 33(1):194200, 2011.

[8] D. R. Hardoon, S. Szedmak, and J. Shawe-Taylor. Canonical correlation analysis: An overview with application to learning methods. Neural computation, 16(12):2639-2664, 2004.

[9] K. Chaudhuri, S. M. Kakade, K. Livescu, and K. Sridharan. Multi-view clustering via canonical correlation analysis. Proceedings of the 26th annual international conference on machine learning, ACM, 129-136, 2009.

[10] X. B. Shen, Q. S. Sun, and Y. H. Yuan. A unified multiset canonical correlation analysis framework based on graph embedding for multiple feature extraction. Neurocomputing, 148:397-408, 2015.

[11] Y. Feng, J. Xiao, Y. Zhuang, and X. Liu. Adaptive unsupervised multiview feature selection for visual concept recognition. Asian Conference on Computer Vision, 343-357, 2012.

[12] H. Wang, F. Nie, and H. Huang. Multi-View Clustering and Feature Learning via Structured Sparsity. Proceedings of the 30th annual international conference on machine learning, 352-360, 2013. 
[13] D. D. Lee, and H. S. Seung. Learning the parts of objects by nonnegative matrix factorization. Nature, 401(6755):788-791, 1999.

[14] S. Z. Li, X. W. Hou, H. J. Zhang, and Q. S. Cheng. Learning spatially localized, parts-based representation. Proceedings of the 2001 IEEE Computer Society Conference on Computer Vision and Pattern Recognition, IEEE, 1(1):I207-I212, 2001.

[15] A. P. Singh, and G. J. Gordon. Relational learning via collective matrix factorization. Proceedings of the 14th ACM SIGKDD international conference on Knowledge discovery and data mining, ACM, 650-658, 2008.

[16] J. Liu, C. Wang, J. Gao, and J. Han. Multi-view clustering via joint nonnegative matrix factorization. SIAM International Conference on Data Mining, 13:252-260, 2013.

[17] S. Y. Zhi, and H. Zhou. Partial multi-view clustering. AAAI Conference on artificial intelligence, 1968-1974, 2014.

[18] D. Cai, X. He, J. Han, and T. S. Huang. Graph regularized nonnegative matrix factorization for data representation. IEEE Transactions on Pattern Analysis and Machine Intelligence, 33(8):1548-1560, 2011.

[19] C. Ding, T. Li, and M. I. Jordan. Convex and semi-nonnegative matrix factorizations. IEEE Transactions on Pattern Analysis and Machine Intelligence, 32(1):45-55, 2010.

[20] C. J. Hsieh, and I. S. Dhillon. Fast coordinate descent methods with variable selection for non-negative matrix factorization. Proceedings of the 17th ACM SIGKDD international conference on Knowledge discovery and data mining, ACM, 1064-1072, 2011.

[21] Z. Lin, M. Chen, and Y. Ma. The augmented lagrange multiplier method for exact recovery of corrupted low-rank matrices. arXiv, arXiv:1009.5055, 2010.

[22] G. Bisson G, and C. Grimal. An architecture to efficiently learn cosimilarities from multi-view datasets. International Conference on Neural Information Processing, Springer Berlin Heidelberg:184-193, 2012.

[23] M. Van Breukelen, R. P. W. Duin, D. M. J. Tax, and J. E. den Hartog. Handwritten digit recognition by combined classifiers. Kybernetika, 34(4):381-386, 1998. 